\title{
Publisher's Note: Limits on Lorentz Invariance Violation from Coulomb Interactions in Nuclei and Atoms [Phys. Rev. Lett. 118, 142501 (2017)]
}

V. V. Flambaum and M. V. Romalis

(Received 10 April 2017; published 20 April 2017)

DOI: 10.1103/PhysRevLett.118.169905

This Letter was published online on 3 April 2017. Modifications are needed in the title and abstract. The title should read as "Limits on Lorentz Invariance Violation from Coulomb Interactions in Nuclei and Atoms." In the abstract the last line should read as "experiments: the speed of light is isotropic to a part in $10^{28}$." The Letter has been corrected as of 10 April 2017. The title and abstract are correct in the printed version of the journal. 